Nouvelles perspectives en sciences sociales

Revue internationale de systémique complexe et d'études relationnelles

\title{
Simulation informatisée en humanités et sciences sociales
}

\section{Enrico Scalas}

Volume 5, numéro 2, mai 2010

Sur le thème de la simulation

URI : https://id.erudit.org/iderudit/044075ar

DOI : https://doi.org/10.7202/044075ar

Aller au sommaire du numéro

\section{Éditeur(s)}

Prise de parole

\section{ISSN}

1712-8307 (imprimé)

1918-7475 (numérique)

Découvrir la revue

\section{Citer cet article}

Scalas, E. (2010). Simulation informatisée en humanités et sciences sociales. Nouvelles perspectives en sciences sociales, 5(2), 59-67.

https://doi.org/10.7202/044075ar

\section{Résumé de l'article}

Ce texte court sur les simulations dans les sciences humaines et sociales est consacré aux simulations multi-agents. Les ingrédients d'une simulation multi-agents sont essentiellement au nombre de deux : agents et interactions. Les Agents avec leurs propriétés interagissent entre eux et les interactions peuvent être soit directes soit indirectes. Par analogie avec les systèmes physiques, la pertinence de l'équilibre statistique en économie peut être aussi analysée. 


\title{
Simulation informatisée en humanités et sciences sociales
}

\author{
EnRICo Scalas \\ Università del Piemonte Orientale
}

T'approche multi-agents employée en économie et en sciences —sociales est de plus en plus populaire chez les auteurs qui veulent poursuivre des recherches innovatrices ${ }^{1}$. Cette approche essaie de réconcilier l'individualisme méthodologique et la présence de phénomènes émergents ${ }^{3}$.

Il existe essentiellement deux composantes aux simulations multi-agents : les agents et les interactions. Les agents, avec les propriétés qui leur sont propres, interagissent les uns avec les autres, soit indirectement, soit directement. Par exemple, nous pouvons observer une interaction indirecte lorsque des agents achètent ou vendent indépendamment les uns les autres des actions sur le marché boursier. Le prix des actions est fixé selon

$1 \quad$ Frédéric Amblard et Denis Phan (dir.), Modélisation et simulation multi-agents, applications pour les Sciences de l'Homme et de la Société, London, Hermes Sciences \& Lavoisier, 2006, p. 446; Leigh Tesfatsion et Kenneth L. Judd (dir.), Handbook of Computational Economics, vol. 2, Agent-Based Computational Economics, Amsterdam, North Holland, 2006.

2 Joseph Schumpeter, Das Wesen und der Hauptinhalt der theoretischen Nationalökonomie, München and Leipzig, Duncker und Humblot, 1908, $626 \mathrm{p}$.

3 Mario Bunge, "Systemism: The Alternative to Individualism and Holism ", Journal of Socio-Economics, no 29, 2000, p. 147-157. 
un mécanisme d'équilibre des marchés même si les agents n'ont pas le droit de communiquer lorsqu'ils inscrivent leurs commandes. Cette situation peut se réaliser en laboratoire, lors d'études sur l'économie behavioriste ${ }^{4}$. Pour bien comprendre les interactions directes, il est aussi possible de puiser des exemples à partir des marchés financiers, notamment par le biais de phénomènes de mimétisme, où un agent-aspirant imite les comportements d'agents reconnus pour leur succès.

Alors que certains de ces concepts peuvent paraître nouveaux, leurs origines philosophiques et scientifiques datent du XIX siècle. À cette époque, l'idée généralement admise était le rapport de ressemblance entre la société des êtres humains, où plusieurs individus interagissaient, et l'univers atmosphérique, où plusieurs atomes ou molécules de gaz faisaient de même. Je nomme ce rapport l'analogie mécanique et on le retrouve, par exemple, dans le livre de Hacking, The Taming of Chance ${ }^{5}$, lorsque l'auteur nous rappelle que Boltzmann l'utilisait pour décrire l'hypothèse atomique.

Une certaine lecture de Guerre et Paix de Tolstoi ${ }^{6}$ nous permet de voir jusqu'à quel point cette analogie avait envahi la pensée de l'époque - non seulement y retrouve-t-on une exploration des comportements et des interactions de 580 personnages durant l'invasion napoléonienne de la Russie, mais, dans le second épilogue du roman, on peut lire :

Speaking of the interaction of heat and electricity and of atoms, we cannot say why this occurs, and we say that it is so because it is inconceivable otherwise, because it must be so and that it is a law.

The same applies to historical events. Why war and revolution occur we do not know. We only know that to produce the one or the other action, people combine in a certain formation in which they all take part, and we say that

\footnotetext{
4 Bence Toth et al., "The Value of Information in a Multi-Agent Market Model. The Luck of the Uninformed ", The European Physical Journal B, $\mathrm{n}^{\circ} 55,2007$, p. 115-120.

5 Ian Hacking, The Taming of Chance, Cambridge (UK), Cambridge University Press, 1990, p. 277.

6 Leo Tolstoï (1869), War and Peace, http://www.gutenberg.org/dirs/etext01/ wrnpc11.txt
} 
this is so because it is unthinkable otherwise, or in other words that it is a law.

Ainsi, l'analogie qui inspire les auteurs actuels en simulations multi-agents était suffisamment populaire au XIX ${ }^{e}$ siècle pour transparaître dans les travaux scientifiques et dans la littérature populaire de l'époque. Très tôt, les études en mécanique statistique ont fait appel à l'analogie mécanique pour justifier l'utilisation du modèle utilisé en physique en sciences humaines.

On retrouve aussi des éléments de l'analogie mécanique dans des recherches récentes en mécanique statistique. L'intérêt de ces recherches est d'avoir inversé l'emploi de l'analogie mécanique, partant du modèle des sciences sociales pour l'appliquer au domaine de la physique. Malheureusement, dans ce cas, les chercheurs ne font que de l'analyse statique des phénomènes à l'étude puisque le concept d'équilibre statistique était inconnu de la plupart d'entre eux durant tout le XIX ${ }^{\mathrm{e}}$ siècle et ce même jusqu'à tout récemment. Dans le but de clarifier ce point, il faut noter que Léon Walras et Vilfredo Pareto ont construit des théories d'équilibre général à partir de l'analogie mécanique, et Ingrao et Israël ${ }^{8}$ confirment l'influence de la physique classique sur le développement du domaine de l'économie. La théorie de l'équilibre général en économie est aussi particulièrement similaire à la théorie de l'équilibre statistique en physique classique. En physique, on minimise une fonction potentielle sujette aux conditions environnantes dans le but de trouver l'équilibre des

7 Traduction de Iouri Petrov, Université d'État de Tomsk, dans « Les raisons anthropologiques de la synthèse analytique dans la connaissance historique », http://ressources-cla.univ-fcomte.fr/gerflint/Russie3/raisons.pdf (consulté le 3 mars 2010). Traduction : «En parlant de l'interrelation de la chaleur et de l'électricité, et en parlant des atomes, nous ne pouvons pas dire, pourquoi cela arrive, et nous disons que cela est ainsi parce qu'autrement cela n'a pas de sens, que cela doit être ainsi, que c'est la loi. / Il en va de même pour ce qui se rapporte aux phénomènes historiques. Pourquoi la guerre ou la révolution? Nous ne le savons pas; nous savons seulement, que pour l'accomplissement de telle ou telle action les gens se constituent une union précise et participent tous; et nous disons que cela est ainsi parce qu'autrement cela n'a pas de sens, que cela doit être ainsi, que c'est la loi.»

8 Bruno Ingrao et Giorgio Israel, The Invisible Hand: Economic Equilibrium in the History of Science, Cambridge (MA), MIT Press, 1990. 
positions d'un système de particules. Dans le but de trouver l'équilibre microéconomique en économie, on maximise la fonction d'utilité d'un individu sujet à des contraintes budgétaires (du côté du consommateur ou de la demande), on maximise les profits de la firme sujette à des contraintes de dépenses (du côté de la production ou de l'offre) et on associe l'offre et la demande pour trouver les quantités équilibrées et les coûts. Dans les deux cas, les outils mathématiques sont les mêmes : l'optimisation avec contraintes, basée sur la méthode du multiplicateur de Lagrange ${ }^{9}$. Le temps n'entre pas explicitement en compte dans la formulation des théories de l'équilibre économique. Le coût et les quantités sont donnés une fois pour toutes. Notre expérience quotidienne montre que les choses ne se passent pas ainsi. Les prix changent dans le temps et dans l'espace. Les firmes et les individus ne sont pas des optimisateurs parfaits ${ }^{10}$.

Le concept d'équilibre statistique diffère du concept d'équilibre mécanique. L'équilibre statistique peut être fondé sur le processus de décision markovien. Si la dynamique d'un système d'agents en interaction est échantillonnée à des intervalles fixes, si l'état de cette dynamique est enregistré et si le nombre d'états est fini, la dynamique peut être décrite par une classe importante du modèle stochastique, notamment les chaînes de Markov finies ${ }^{11}$. Ces processus sont entièrement caractérisés par deux distributions de probabilité : la probabilité initiale et la probabilité de transition. La probabilité initiale accorde un poids initial aux états du système. Si le système se trouve dans un état donné, son poids est 1 , alors que tous les autres états ont un poids équivalent à 0 . La probabilité en transition donne la probabilité que le système

$9 \quad$ I. B. Vapnyarskii, « Lagrange Multipliers », dans Michiel Hazewinkel (dir.), Encyclopaedia of Mathematics, Kluwer Academic Publishers, 2001, site : http://eom.springer.de/ (article "Lagrange Multiplier", accessible aussi sur http://eom.springer.de/L/1057190.htm), consulté le 5 mars 2010.

10 Herbert Simon, Models of Bounded Rationality, vol. 1, MIT Press, Cambridge (MA), 1982; Models of Bounded Rationality, vol. 2, Cambridge (MA) MIT Press, 1982; Models of Bounded Rationality, vol. 3, Cambridge (MA), MIT Press, 1997.

11 John G Kemeny et J. Laurie Snell, Finite Markov Chains, Van Nostrand, New Jersey, Princeton, 1960. 
actuellement dans l'état $i$, se retrouve à l'état $j$ lors de la prochaine observation, pour chaque couple d'états. Si les conditions propices sont satisfaites, après un certain moment, le comportement de la chaîne de Markov est finalement décrit comme une distribution de probabilités invariantes. Cela veut dire que la probabilité de tous les états du système ne change plus. Notez bien que cela ne veut pas dire que l'état du système ne change plus, comme c'est le cas avec l'équilibre mécanique. Bien au contraire, l'équilibre statistique est dynamique : le système est en mouvement constant, d'un état à un autre Mais si vous comptez la fréquence relative avec laquelle ces états apparaissent, la distribution de probabilité reste invariable ${ }^{12}$.

Un nombre important de modèles multi-agents en sciences sociales et en économie sont déjà des chaînes de Markov. Ainsi, le concept d'équilibre statistique présenté ci-dessus est utile lorsque nous tentons de décrire les modèles d'un point de vue théorique. Toutefois, la question consiste à savoir si l'équilibre statistique est vraiment utile en économie (ou dans d'autres domaines des sciences sociales).

Certes, il est possible de justifier la pertinence de l'équilibre statistique dans tous les domaines des sciences, de manière générale. Lorsque nous décrivons la réalité qui nous entoure, nous commençons en classant les objets en classes/en catégories et nous énumérons les objets de toutes les catégories. Ensuite, certains objets changent et le temps est utilisé comme un outil (label) pour décrire le changement. De plus, le changement indique que l'objet se déplace d'une catégorie à l'autre. Ce changement peut s'expliquer par la chaîne de Markov si nous ne connaissons pas les détails du mouvement (et certains penseurs croient même que nous ne connaîtrons jamais ces détails). Toujours dans cette même optique, il est possible de dériver des

12 Richard von Mises, "Wahrscheinlichkeitsrechnung und ihre Anwendung ", dans Der Statistik und theoretischen Physik, New York, Rosenberg, 1945; Oliver Penrose, Foundations of Statistical Mechanics: A Deductive Treatment, New York, Dover, 1970; Ubaldo Garibaldi et Enrico Scalas, Finitary Probabilistic Methods in Econophysics, forthcoming, Cambridge (UK), Cambridge University Press, 2010. 
modèles de diffusion comme des limites discrètes des chaînes de naissance-mortalité de Markov. Si les chaînes de naissancemortalité de Markov ne sont pas suffisantes pour décrire le changement, à cause d'effets d'espace et de mémoire non locaux, nous sommes naturellement menés vers des modèles à anomalies diffusives.

La pertinence de l'équilibre statistique en économie et en sciences sociales a été contestée par au moins deux points de vue $^{13}$. Afin de ne pas m'éterniser sur le sujet, je vais me limiter au domaine de l'économie. Certains penseurs ${ }^{14}$ ne croient pas que les systèmes économiques peuvent atteindre un certain équilibre, ou ne peuvent pas atteindre un équilibre statistique, puisque, à leur avis, de tels systèmes sont fortement déséquilibrés. D'aucuns, notamment les économistes post-keynesiens, contestent l'idée que le comportement des systèmes macroéconomiques puisse être expliqué en termes d'interactions individuelles. En d'autres mots, ils contestent la fondation micro du macroéconomique, pouvant inclure une micro-fondation probabiliste en termes d'équilibre statistique. Ma réponse à ces objections est que le concept d'équilibre statistique pourrait servir même en économie et pourrait être utile pour décrire et justifier des propriétés distribuées empiriquement. C'est donc dire qu'avant même de réfuter le concept, il faudrait en explorer les conséquences et comprendre ce qui se passe à l'intérieur de modèles simples. Il est tout à fait possible que les dynamiques économiques soient suffisamment rapides pour atteindre un équilibre statistique même en présence du système dit équilibré ${ }^{15}$. Une autre objection courante en économie est que le modèle d'équilibre statistique ne tient pas compte de variables pertinentes, comme le niveau de vie ou le nombre de travailleurs. Il est vrai que certaines

13 Mauro Gallegatia et al., "Worrying Trends in Econophysics ", Physica A, $\mathrm{n}^{\circ} 370,2006$, p. 1-6.

14 Joseph I. McCauley, «Thermodynamic Analogies in Economics and Finance: Instability of Markets ", Physica A, no 329, 2003, p. 199-212.

15 Peter Richmond, Bikas K. Chakrabarti, Chatterjee Arnab et John Angle, "Comments on "Worrying Trends in Econophysics": Income Distribution Models ", dans Arnab Chatterjee et Bikas K. Chakrabarti (dir.), Econophysics of Stock and other Markets, New York, Springer, 2006, p. 244-253. 
recherches ignorent ces composantes économiques. Toutefois, certaines études en tiennent compte, et ce, en appliquant le concept d'équilibre statistique ${ }^{16}$. Il faut donc comprendre que l'absence de certaines variables n'est pas un obstacle majeur. Finalement, une dernière objection ne nie pas la pertinence de l'équilibre statistique, mais ne comprend pas l'avantage de la description des dynamiques probabilistes. Lorsqu'on considère de grands agrégats macroéconomiques ou une longue évolution d'un système, les fluctuations pourraient devenir non pertinentes. Dans ces cas, ce sont seulement les descriptions de dynamiques probabilistes qui maintiennent leur importance - les processus stochastiques pouvant être remplacés par des différences de moyenne ou des équations différentielles déterminant celles-ci. En réponse à cette objection, on doit considérer le système auto-moyennant (lack of self averaging ${ }^{17}$ ) qui est souvent là, en présence de corrélations fortes. Un système auto-moyennant s'observe lorsque le ratio entre les fluctuations autour des moyennes et les moyennes elles-mêmes disparaissent au cours du temps du fait de l'agrégation. Toutefois, les corrélations fortes montrent que le ratio ne disparaît pas du tout; le concept d'équilibre statistique, par le biais des processus stochastiques, est donc nécessaire à l'étude de ces cas en particulier.

16 Voir Ubaldo Garibaldi et Enrico Scalas, Finitary Probabilistic Methods in Econophysics..., op. cit.

17 Masanao Aoki and Hiroshi Yoshikawa, « Non-Self-Averaging in Macroeconomic Models: A Criticism of Modern Micro-Founded Macroeconomics ", Economics Discussion Papers, no 2007-49; http://www.economics-ejournal.org/economics/discussionpapers/2007-49. 


\section{Bibliographie}

Amblard, Frédéric et Denis Phan (dir.), Modélisation et simulation multiagents, applications pour les Sciences de l'Homme et de la Société, London, Hermes Sciences \& Lavoisier, 2006.

Aoki, Masanao et Hiroshi Yoshikawa, " Non-Self-Averaging in Macroeconomic Models: A Criticism of Modern Micro-founded Macroeconomics ", Economics Discussion Papers, no 2007-49, http:// www.economics-ejournal.org/economics/discussionpapers/2007-49.

Bunge, Mario, «Systemism: The Alternative to Individualism and Holism ", Journal of Socio-Economics, n² 29, 2000, p. 147-157.

Gallegatia, Mauro, Steven Keen, Thomas Lux et Paul Ormerod, «Worrying Trends in Econophysics ", Physica A, n 370, 2006, p. 1-6.

Garibaldi, Ubaldo et Enrico Scalas, Finitary Probabilistic Methods in Econophysics, forthcoming, Cambridge (UK), Cambridge University Press, 2010.

Hacking, Ian, The Taming of Chance, Cambridge (UK), Cambridge University Press, 1990.

Ingrao, Bruno et Giorgio Israel, "The Invisible Hand: Economic Equilibrium in the History of Science », Cambridge (MA), MIT Press, 1990.

Kemeny, John G. et J. Laurie Snell, Finite Markov Chains, Van Nostrand, New Jersey, Princeton, 1960.

McCauley, Joseph I., "Thermodynamic Analogies in Economics and Finance: Instability of Markets ", Physica A, n ${ }^{\circ} 329,2003$, p. 199212.

Penrose, Oliver, Foundations of Statistical Mechanics: A Deductive Treatment, New York, Dover, 1970.

Richmond, Peter, Bikas K. Chakrabart, Arnab Chatterjee et John Angle, "Comments on "Worrying Trends in Econophysics": Income Distribution Models ", dans Arnab Chatterjee et Bikas K. Chakrabarti (dir.), Econophysics of Stock and Other Markets, New York, Springer, 2006, p. 244-253.

Schumpeter, Joseph, Das Wesen und der Hauptinhalt der theoretischen Nationalökonomie, München and Leipzig, Duncker und Humblot, 1908.

Simon, Herbert A., Models of Bounded Rationality, vol. 1, Cambridge (MA), MIT Press, 1982.

Simon, Herbert A., Models of Bounded Rationality, vol. 2, Cambridge (MA), MIT Press, 1982. 
Simon Herbert A., Models of Bounded Rationality, vol. 3, Cambridge (MA), MIT Press, 1997.

Tesfatsion, Leigh et Kenneth L. Judd (dir.), Handbook of Computational Economics, vol. 2, Agent-Based Computational Economics, Amsterdam, North Holland, 2006.

Toth, Bence, Enrico Scalas, Jürgen Huber et Michael Kirchler, «The Value of Information in a Multi-Agent Market Model. The Luck of the Uninformed ", The European Physical Journal B, n ${ }^{\circ} 55,2007$, p. 115120.

Tolstoy, Leo N. (1869), War and Peace, http://www.gutenberg.org/dirs/ etext01/wrnpc11.txt, traduction de Iouri Petrov, Université d'Etat de Tomsk, dans "Les raisons anthropologiques "de la synthèse analytique" dans la connaissance historique ", http://ressources-cla.univ-fcomte.fr/ gerflint/Russie3/raisons.pdf, consulté le 3 mars 2010.

Vapnyarskii, I.B., "Lagrange Multipliers », dans Michiel Hazewinkel (dir.), Encyclopaedia of Mathematics, Kluwer Academic Publishers, 2001, site : http://eom.springer.de/ (article "Lagrange Multiplier", accessible aussi sur http://eom.springer.de/L/1057190.htm), consulté le 5 mars 2010.

Mises, Richard von, "Wahrscheinlichkeitsrechnung und ihre Anwendung », dans Der Statistik und theoretischen Physik, New York, Rosenberg, 1945. 